Onkologe 2022 28 (Suppl 1):S2-S3

https://doi.org/10.1007/s00761-022-01103-0 Angenommen: 20. Januar 2022

(๑) The Author(s), under exclusive licence to Springer Medizin Verlag GmbH, ein Teil von Springer Nature 2022

\title{
Klinische Forschung in der Onkologie - Studiennetzwerke als Motor in der Forschung
}

\author{
Eray Gökkurt ${ }^{1}$ Emmanouil Fokas ${ }^{2}$. Pompiliou Piso ${ }^{3} \cdot$ Anke Reinacher-Schick $^{4}$ \\ ${ }^{1}$ Hämatologisch-Onkologische Praxis Eppendorf (HOPE), Hamburg, Deutschland \\ ${ }^{2}$ Klinik für Strahlentherapie und Onkologie, Universitätsklinikum Frankfurt, Frankfurt, Deutschland \\ ${ }^{3}$ Klinik für Allgemein- und Viszeralchirurgie, Krankenhaus Barmherzige Brüder Regensburg, Regensburg, \\ Deutschland \\ ${ }^{4}$ St. Josef-Hospital, Klinik für Hämatologie und Onkologie mit Palliativmedizin, Klinikum der Ruhr- \\ Universität, Bochum, Deutschland
}

Liebe Kolleginnen und Kollegen,

in dieser turbulenten noch immer pandemiegeprägten Zeit freuen wir uns über die großartige Möglichkeit, mit diesem Sonderheft unsere gemeinsame Studienarbeit, aber auch weitere Themen der Arbeitsgemeinschaft Internistische Onkologie (AIO) in Kooperation mit der Assoziation Chirurgische Onkologie (ACO) und der Arbeitsgemeinschaft Radiologische Onkologie (ARO) bekannt zu machen und alle Interessierten dafür zu begeistern. An dieser Stelle möchten wir allen Autor*innen, die die vielfältigen Beiträge und Themen koordiniert und den Wissensextrakt für eine erkenntnisreiche Lektüre zusammengefasst haben, ausdrücklich für ihr Engagement danken.

Insgesamt bietet dieses Sonderheft einen spannenden Überblick über die Bandbreite translationaler und klinischer Studien in der Internistischen Onkologie in Kooperation mit der Chirurgie, der Radioonkologie und weiteren Partnergruppen.

Den Herausforderungen und Perspektiven der Präzisionsonkologie haben wir einen eigenen Artikel gewidmet. Wie von Professorin Sonja Loges aus Mannheim/ Heidelberg dargestellt, stehen wir hierzulande vor erheblichen strukturellen und logistischen Herausforderungen, die es zu bewältigen gilt, um weiterhin bestmögliche Patient:innenversorgung zu ermöglichen und gleichzeitig an Wahrnehmung als Innovationsstandort im internationalen Wettbewerb weiter zu gewinnen. In Bezug auf die Präzisionsonkologie bedeutet dies insbesondere eine Optimierung der Logistik im Bereich der Durchführung früher klinischer Studien, um unseren Patient:innen möglichst frühzeitig Zugang zu innovativen Therapeutika zu ermöglichen. Hier ist es uns eine besondere Freude, dass wir mit Frau Söhlke und Herrn Wartenberg ausgewiesene Patient:innenvertreter als Koautor*in zum Thema gewinnen konnten.

Zudem ist die Durchführung klinischer Studien mit regulativen Vorgaben verknüpft, die zunehmend in, nicht immer zielführende, bürokratische Anforderungen münden und Prozesse deutlich verlangsamen. Hierzu erfolgte während der AIO-Herbsttagung 2020 eine virtuelle Expert:innendiskussion von Stakeholdern, die typische Hürden in der raschen Umsetzung von klinischen Studien identifizieren konnten. Der Beitrag "Bürokratieabbau in klinischen Studien", federführend von Prof. Grünwald aus Essen zusammengebracht, beleuchtet und analysiert diese Konflikte und zeigt gleichzeitig realistische Lösungsvorschläge auf.

\section{\) Die Förderung des wissen- schaftlichen Nachwuchses in der Onkologie ist eines unserer zentralen Themen}

Die Förderung des wissenschaftlichen Nachwuchses in der Onkologie ist eines unserer zentralen Themen. Das wissenschaftliche Potenzial unserer jungen Kolleg:innen zu erkennen, zu fördern und zu fordern, sie aber auch eng in Entscheidun- 
Infobox 1

Dieses Sonderheft erscheint in Kooperation mit der

Arbeitsgemeinschaft Internistische Onkologie (AIO)

Die Arbeitsgemeinschaft Internistische Onkologie in der Deutschen Krebsgesellschaft e.V. (AIO) wurde 1976 gegründet und zählt heute ca. 1400 ordentliche, außerordentliche, assoziierte und fördernde Mitglieder, die in mehr als 20 Arbeitsgruppen ehrenamtlich tätig sind. Ihre wichtigsten Aufgaben sieht die AIO in der Durchführung von klinischen Studien und in der Sicherung und Optimierung der Qualität der medikamentösen Tumortherapie.

www.aio-portal.de

Assoziation Chirurgische Onkologie (ACO)

Die Assoziation Chirurgische Onkologie wurde auf der Viszeralmedizin 2017 in Dresden gemeinsam mit den Arbeitsgemeinschaften der DGAV gegründet. Ihr Ziel ist es, das wichtige Gebiet der chirurgischen Onkologie innerhalb der Allgemein- und Viszeralchirurgie zu stärken und dessen Bedeutung für das Fachgebiet zu betonen.

Die Einführung eines national und international abgestimmten Curriculums Chirurgische Onkologie mit einer entsprechenden Spezialisierung war eine der wichtigsten Errungenschaften der ACO. Die zentrale Bedeutung der hohen Operationsqualität sowie der interdisziplinär abgestimmten multimodalen Therapie entspricht dem Credo der ACO. Gerade für innovative Behandlungskonzepte oder Operationstechniken ist für die ACO die Behandlung innerhalb von Studien essenziell.

www.aco-chirurgie.de

Arbeitsgemeinschaft Radiologische Onkologie (ARO)

Die Arbeitsgemeinschaft Radiologische Onkologie arbeitet an der Optimierung und

Weiterentwicklung der Radiotherapie für die Behandlung von malignen Erkrankungen.

Eine der höchsten Prioritäten der ARO ist die Nachwuchsförderung. Seit 2017 ist das

Mentoringprogramm junger Mediziner zur Erstellung klinischer Studienprotokolle etabliert. Dieses erfolgt in enger Zusammenarbeit mit der Deutschen Gesellschaft für Radioonkologie (DEGRO) und deren Nachwuchsorganisation (jDEGRO). Einmal jährlich wird der ARO-Studienpreis für die beste Studie einer jungen Studienautorin/eines jungen Studienautors vergeben. Als kompetente Ansprechpartnerin steht sie zur Verfügung, um interdisziplinäre Leitlinien zu verfassen bzw. zu aktualisieren und um neue klinische Studienprotokolle auszuarbeiten.

www.aro-online.de

AIO, ACO und ARO gehören zu den 25 Arbeitsgemeinschaften der Deutschen Krebsgesellschaft e. V. (DKG)

Die DKG - eine Nachfolgeorganisation des 1900 gegründeten "Comité für Krebssammelforschung" - ist die größte wissenschaftlich-onkologische Fachgesellschaft im deutschsprachigen Raum. In der DKG vertreten sind rund 8000 Einzelmitglieder in 25 Arbeitsgemeinschaften, die sich mit der Erforschung und Behandlung von Krebserkrankungen befassen; dazu kommen 16 Landeskrebsgesellschaften und 36 Fördermitglieder. Die DKG engagiert sich für eine Krebsversorgung auf Basis von evidenzbasierter Medizin, Interdisziplinarität und konsequenten Qualitätsstandards, ist Mitinitiatorin des Nationalen Krebsplans und Partnerin der "Nationalen Dekade gegen Krebs". www.krebsgesellschaft.de

gen einzubinden, diesem Anliegen wollen wir nicht nur heute, sondern auch in $\mathrm{Zu}$ kunft Rechnung tragen. Wir freuen uns hier besonders, dass unsere jungen Onkolog:innen mit erfolgreich durchgeführten Projekten, wie dem MentorUs-Programm, aber auch eigenen Studien, Flagge zeigen konnten und sich als eigene, innovative Arbeitsgruppe darstellen.

Der Beitrag (Federführung Dr. Stahler, Dr. Heinrich) konnte erfreulicherweise in Abstimmung mit den Kolleg:innen der Jungen Chirurgie (als Vertreter der ACO) sowie der Jungen DEGRO (als Vertreter der ARO) erarbeitet werden, was die Bedeutung des interdisziplinären Austauschs bereits früh in der Ausbildung und wissenschaftlichen Karriere betont und aufgreift.
Die Kooperation mit dem Berufsverband der Niedergelassenen Hämatologen und Onkologen (BNHO) als berufspolitischer Zusammenschluss der Kolleg:innen aus den Schwerpunktpraxen, die herausragende Strukturen bei Patientenversorgung und Forschungsaktivität aufweisen, konnte von beiden Seiten weiter gestärkt werden. Wir freuen uns, dass wir gemeinsam mit Prof. Knauf (BNHO-Vorsitzender) und PD Dr. Illmer (stellvertretender BNHO-Vorsitzender) die Gelegenheit nutzen können, die Kooperation der AIO mit dem BNHO mit einem eigenen Beitrag in diesem Sonderheft vorzustellen.

In der Praxis werden Therapieentscheidungen zunehmend komplexer und bedürfen einer stetigen interdisziplinären Abstimmung. Es ist naheliegend, dass auch in der Studienentwicklung und -durchführung die Expertise aller beteiligten Disziplinen gleichermaßen gefordert ist. Den weitaus größten Teil nehmen daher die Beiträge zu unseren laufenden und geplanten Studien ein, die gemeinsam mit den Kolleg:innen der Strahlentherapie und Chirurgie in kollektiver Zusammenarbeit erstellt worden sind.

Das Rektumkarzinom gilt als eines der Musterbeispiele für den Erfolg der interdisziplinären Zusammenarbeit der ACO, der ARO und der AIO. Wir würden uns wünschen, dass wir diejenigen, die sich bisher noch wenig mit dem Thema klinische Forschung befasst haben, künftig als engagierte Mitstreiter*innen gewinnen können. Den Experten der Materie hoffen wir mit dem vorliegenden Sonderheft "Studien" einen aktuellen Überblick der AIO in Kooperation mit den Kolleg:innen der ACO und ARO an die Hand zu geben. Gerade im klinischen Alltag in der Behandlung unserer Patient*innen begegnen uns Fragen, die man offen stellen und diskutieren sollte; entwickeln sich doch daraus spannende Forschungsprojekte, die viele unserer drängenden Fragen heute und in Zukunft beantworten.

Für Anregungen, konstruktive Kritik, aber auch für Ihre Fragen sind wir jederzeit offen. Zögern Sie nicht, uns zu kontaktieren!

Ihre

Prof. Dr. Anke Reinacher-Schick

Vorsitzende der Arbeitsgemeinschaft Internistische Onkologie (AIO) in der Deutschen Krebsgesellschaft e.V. (DKG) als federführende Herausgeberin im Namen aller Mitautoren

\section{Korrespondenzadresse}

\section{Prof. Dr. Anke Reinacher-Schick}

St. Josef-Hospital, Klinik für Hämatologie und Onkologie mit Palliativmedizin, Klinikum der Ruhr-Universität

Gudrunstraße 56, 44791 Bochum, Deutschland reinacher@aio-portal.de

Interessenkonflikt. E. Gökkurt, E. Fokas, P. Piso und A. Reinacher-Schick geben an, dass kein Interessenkonflikt besteht. 